\title{
A Case of De Novo Psoriasis Secondary to Nivolumab in a Patient With Metastatic Renal Cell Carcinoma
}

\author{
Sanjana Mullangi ${ }^{1}$, Sreeja Ponnam ${ }^{2}$, Manidhar Reddy Lekkala ${ }^{3}$, Supriya Koya ${ }^{4}$ \\ 1. Internal Medicine, Hillcrest Medical Center, Tulsa, USA 2. Neurosurgery, Oklahoma Surgical Hospital, Tulsa, USA 3. \\ Hematology and Oncology, University of Rochester Medical Center, Rochester, USA 4. Hematology and Oncology, \\ Hillcrest Medical Center, Tulsa, USA
}

Corresponding author: Sanjana Mullangi, sanjana.mullangi.md@gmail.com

\begin{abstract}
Immune-mediated adverse events are commonly seen with immune checkpoint inhibitors like nivolumab. Oncology specialists usually have to screen patients for risk factors for autoimmune diseases, since immune checkpoint inhibitors can potentially exacerbate these events. Some of the immune-mediated side effects include polyneuropathies, colitis, and cutaneous adverse effects. Non-specific maculopapular rash, pruritus, lichenoid reactions, eczema, and vitiligo are the most common dermatologic side effects. It is thought that these adverse events are due to the blocking of the programmed cell death protein-1 (PD-1) pathway and are mediated by the cytotoxic T cells. Psoriasis has been previously reported as a side effect in a few case reports and most commonly presented as an exacerbation of preexisting psoriasis. However, de novo psoriasis occurrence as a result of nivolumab is a rare entity, especially in a non-melanoma patient. Here, we present a case of renal cell carcinoma treated with immunotherapy with nivolumab, who developed de novo psoriasis with palmoplantar involvement.
\end{abstract}

Categories: Internal Medicine, Oncology

Keywords: nivolumab, psoriasis, de novo psoriasis, renal cell carcinoma, pd-1 inhibitors, immune check-point inhibitor, drug-induced psoriasis, immune mediated side effects

\section{Introduction}

Programmed cell death protein 1 (PD-1) is considered to be an immune checkpoint molecule. Immune checkpoint inhibitors block the interaction between PD-1 and PD ligand-1, stimulating the T-cell activity and helping the anticancer host immune response [1]. Nivolumab is an IgG4 anti-PD-1 monoclonal antibody, which belongs to the class of immune checkpoint inhibitors and is approved in various cancers, including renal cell carcinoma [2]. The blockage of the PD-1 pathway is thought to cause immune-mediated adverse events such as organ-specific side effects and cutaneous side effects [3]. There are reports of anti-PD-1 induced de-novo psoriasis and exacerbation of previous psoriasis. De-novo psoriasis is usually seen in melanoma patients treated with anti-PD-1 inhibitors [4]. We report a case of a de-novo moderate to severe palmoplantar pustular psoriasis as a side effect from nivolumab in a renal cell carcinoma patient.

Review began 03/31/2021 Review ended 04/19/2021 Published 06/16/2021

(c) Copyright 2021 Mullangi et al. This is an open access article distributed under the terms of the Creative Commons Attribution License CC-BY 4.0., which permits unrestricted use, distribution, and reproduction in any medium, provided the original author and source are credited.

\section{Case Presentation}

A 66-year-old male with a past medical history of metastatic renal cell carcinoma presented with progressive rash while on nivolumab. He was initially diagnosed with Stage III renal cell carcinoma four years ago and underwent nephrectomy. Post-surgery, he was noted to have thyroid nodules on his scans, the biopsy of which indicated metastatic renal cell carcinoma. He underwent thyroidectomy and was started on pazopanib $800 \mathrm{mg}$ daily two months after the surgery for his favorable risk renal cell carcinoma, which required dose adjustment due to side effects. A year later, he was noted to have a growing nodule in the right lower lung and underwent wedge resection. The biopsy showed a metastatic renal cell carcinoma. He was subsequently treated with axitinib and cabozantinib for the progression of the disease. However, he was switched to nivolumab seven months prior to presentation due to the progression of the disease. He tolerated the medication well before he developed progressive symptoms of rash on his palms, arms, and feet. He was evaluated by dermatology for his bright red, symmetrical, round, scaly, crusted, fleshy plaques and was diagnosed with palmoplantar psoriasis. He was started on topical steroids with triamcinolone, which didn't help his symptoms, and needed apremilast and retinoids. Nivolumab was continued through these symptoms, but three months later, he developed severe diarrhea, requiring systemic steroids and infliximab. Nivolumab was held at the time. The repeat surveillance scans did not show any evidence of disease, and he continued to stay off therapy without recurrence of disease at his last follow-up two years after stopping nivolumab. Note the improvement noted in Figure 1 and Figure 2. 


\section{Cureus}

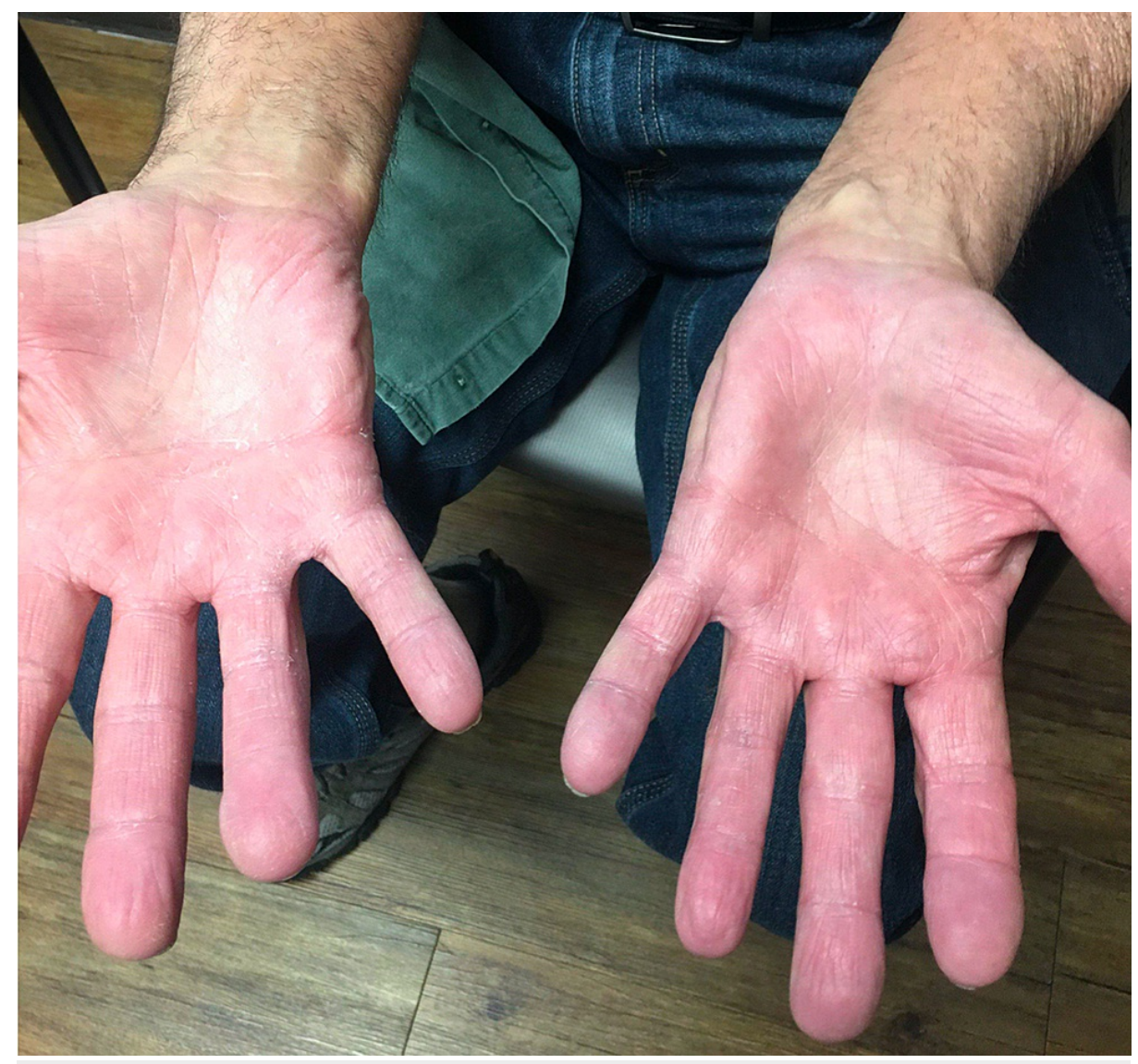

FIGURE 1: Showing improvement in the palmar psoriatic changes (Notice subtle symmetrical erythematous lesions with scaly plaques).

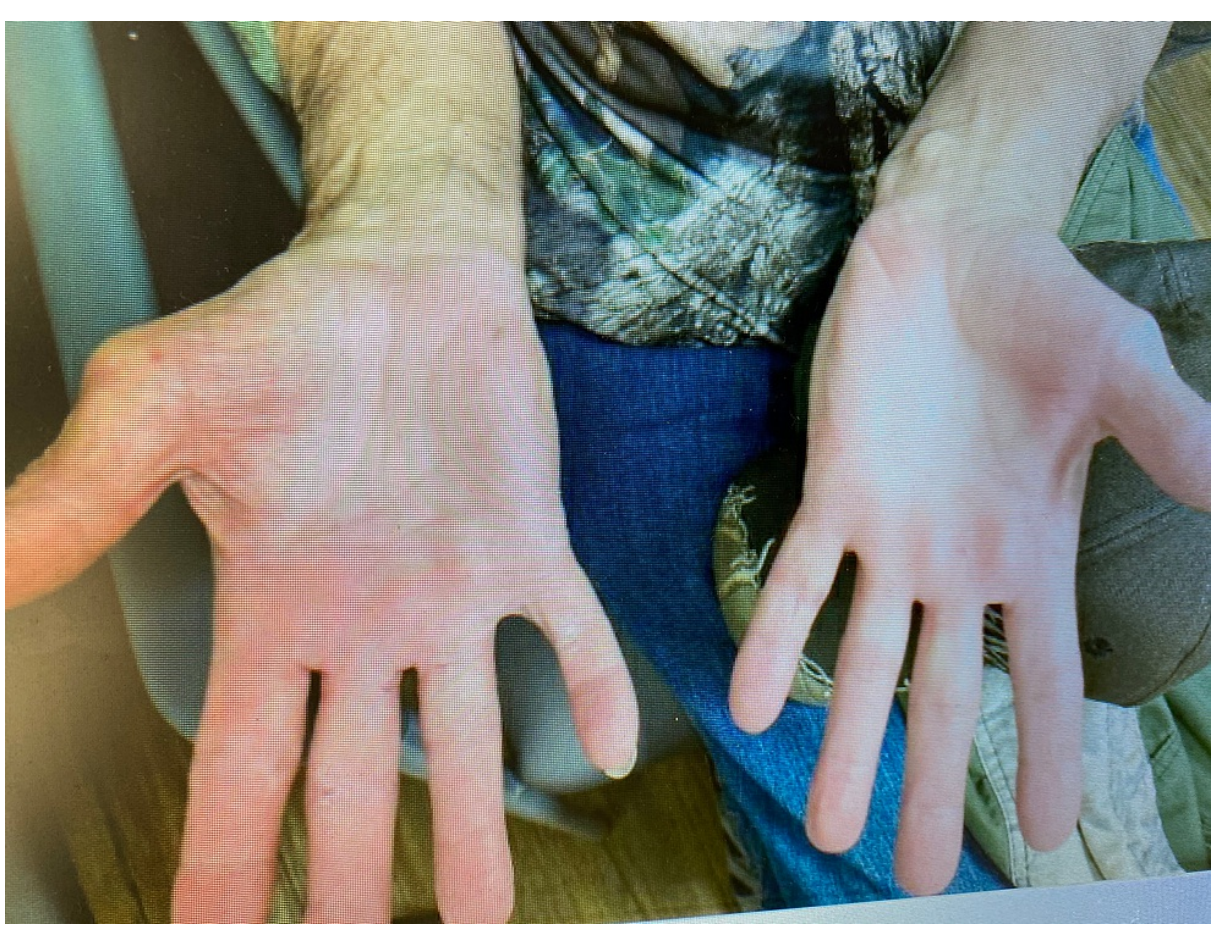

FIGURE 2: Notice further improvement from Figure 1, with almost baseline palmar surface. 


\section{Discussion}

Checkpoint inhibitors are being widely used, and their indications have been increasing. These medications are known to cause immune-mediated side effects and cutaneous toxicities frequently. Non-specific maculopapular rash, pruritus, lichenoid reactions, eczema, and vitiligo are the most common dermatologic side effects [5]. Psoriasis has been previously reported as a side effect in a few case reports and most commonly presented as an exacerbation of preexisting psoriasis [6]. The occurrence of de-novo psoriasis as a result of nivolumab is rare, especially in a non-melanoma patient but few cases were reported $[7,8]$.

In patients with melanoma, the occurrence of psoriasis is thought to be due to the upregulation of metalloproteinase with thrombospondin motif-like 5 (ADAMTSL5) and disintegrin. The ADAMTSL5 specific $\mathrm{CD} 8+\mathrm{T}$ cells are thought to trigger psoriasis, although this scenario is not applicable in non-melanotic cases [9]. It is known that PD-1 blockade augments the Th1 and Th17 responses, which correlate with the antitumor effect [10]. IL-17, the principal cytokine from Th17 cells, plays a key role in the pathogenesis of psoriasis. Furthermore, it is noted that PDL-1 is expressed in 95 percent of IL-17 producing T cells in psoriatic skin, which are activated by nivolumab [11].

There is a delay between the initiation of the anti-PD-1 and the development of psoriasis as seen in our patient. The delay is higher for de-novo psoriasis compared to exacerbation of underlying psoriasis [6]. Psoriasis is characterized by a wide range of clinical manifestations from mild to severe forms. Most of the patients exhibit asymptomatic, psoriasiform lesions, and immunotherapy can be continued in most cases. Psoriasis can be treated with vitamin D, topical steroids, oral steroids, and retinoids based on the severity of the lesions $[5,6]$. Less commonly, there are reports of palmoplantar involvement as was seen in our case.

\section{Conclusions}

We report a rare case of de novo palmoplantar psoriasis occurring as a side effect of nivolumab treatment in a patient with metastatic renal cell carcinoma. Before initiating these immunotherapy drugs, patient history should be carefully checked for both family and personal immune-related diseases. Early recognition of side effects and prompt institution of appropriate immunosuppressive treatment can help continue checkpoint inhibitor therapy without interruption.

\section{Additional Information \\ Disclosures}

Human subjects: All authors have confirmed that this study did not involve human participants or tissue. Conflicts of interest: In compliance with the ICMJE uniform disclosure form, all authors declare the following: Payment/services info: All authors have declared that no financial support was received from any organization for the submitted work. Financial relationships: All authors have declared that they have no financial relationships at present or within the previous three years with any organizations that might have an interest in the submitted work. Other relationships: All authors have declared that there are no other relationships or activities that could appear to have influenced the submitted work.

\section{References}

1. Medina PJ, Adams VR: PD-1 pathway inhibitors: immuno-oncology agents for restoring antitumor immune responses. Pharmacotherapy. 2016, 36:317-34. 10.1002/phar.1714

2. Motzer RJ, Escudier B, McDermott DF, et al.: Nivolumab versus everolimus in advanced renal-cell carcinoma. N Engl J Med. 2015, 373:1803-13. 10.1056/NEJMoa1510665

3. Vivar KL, Deschaine M, Messina J, et al.: Epidermal programmed cell death-ligand 1 expression in TEN associated with nivolumab therapy. J Cutan Pathol. 2017, 44:381-4. 10.1111/cup.12876

4. Kato Y, Otsuka A, Miyachi Y, Kabashima K: Exacerbation of psoriasis vulgaris during nivolumab for oral mucosal melanoma. J Eur Acad Dermatol Venereol. 2016, 30:e89-91. 10.1111/jdv.13336

5. Sibaud V, Meyer N, Lamant L, Vigarios E, Mazieres J, Delord JP: Dermatologic complications of anti-PD1/PD-L1 immune checkpoint antibodies. Curr Opin Oncol. 2016, 28:254-63. 10.1097/CCO.0000000000000290

6. Bonigen J, Raynaud-Donzel C, Hureaux J, et al.: Anti-PD1-induced psoriasis: a study of 21 patients . J Eur Acad Dermatol Venereol. 2017, 31:e254-7. 10.1111/jdv.14011

7. Guven DC, Kilickap S, Guner G, Taban H, Dizdar O: Development of de novo psoriasis during nivolumab therapy in a patient with small cell lung cancer. J Oncol Pharm Pract. 2020, 26:256-8. $10.1177 / 1078155219877234$

8. Law-Ping-Man S, Martin A, Briens E, Tisseau L, Safa G: Psoriasis and psoriatic arthritis induced by nivolumab in a patient with advanced lung cancer. Rheumatology. 2016, 55:2087-9. 10.1093/rheumatology/kew281

9. Nonomura Y, Otsuka A, Ohtsuka M, Yamamoto T, Dummer R, Kabashima K: ADAMTSL5 is upregulated in melanoma tissues in patients with idiopathic psoriasis vulgaris induced by nivolumab. J Eur Acad Dermatol Venereol. 2017, 31:e100-1. 10.1111/jdv.13818

10. Ohtsuka M, Miura T, Mori T, Ishikawa M, Yamamoto T: Occurrence of psoriasiform eruption during nivolumab therapy for primary oral mucosal melanoma. JAMA Dermatol. 2015, 151:797-9. 10.1001/jamadermatol.2015.0249

11. Kamiyama T, Watanabe H, Iijima M, Miyazaki A, Iwamoto S: Coexpression of CCR6 and CD146 (MCAM) is a 


\section{Cureus}

marker of effector memory T-helper 17 cells. J Dermatol. 2012, 39:838-42. 10.1111/j.13468138.2012.01544.x 